\title{
Swept-source OCT with coherent imaging fibre bundles
}

\author{
Helen D Ford ${ }^{*}$ and Ralph P Tatam \\ Engineering Photonics Group, Cranfield University, Cranfield, Bedford, MK43 0AL, UK
}

\begin{abstract}
OCT probes for endoscopy typically use a single optical fibre to deliver light to the measurement region. 2D imaging is achieved using mechanical scanning components at the probe tip.

We are investigating an OCT system in which fibre imaging bundles replace the single probe fibre, allowing many thousand measurement points to be addressed without any mechanical scanning at the probe tip, over a region measuring a few millimetres in each dimension. This allows a small-diameter, electrically passive probe to be engineered using offthe-shelf scanning optics. Images from a single-fibre, conventionally-scanned probe and from a bundle-based probe are presented, using swept-source processing centred at $1330 \mathrm{~nm}$.
\end{abstract}

Keywords: OCT, optical coherence tomography, coherent fibre bundles, imaging bundles, swept-source

\section{INTRODUCTION}

Current endoscopic optical coherence tomography (OCT) probes comprise a single fibre mounted in an external housing ${ }^{1,2}$. Lenses focus the output beam from the delivery fibre onto the sample of interest, providing an OCT measurement at a single point, and the beam is scanned in one or two dimensions to address a line or area of sampling points within the region of interest. The use of rotating components within the endoscope tip provides imaging over an arc centred on the probe, which is particularly suitable for looking at the internal surfaces of cylindrical samples ${ }^{3}$. Implementations involving miniature scanners or MEMS devices have also been demonstrated, which commonly address a rectangular region of the sample surface ${ }^{4}$.

A coherent imaging fibre bundle is an ordered arrangement of small-diameter fibre cores, each of which occupies the same relative position in the surrounding matrix at either end of the bundle. Imaging fibre bundles typically contain 10,000-100,000 fibres with core diameters ranging from a few micrometres to a few tens of micrometres. An image projected onto one end of the bundle is transmitted to the opposite end without distortion.

We have previously investigated the use of bundles in a time-domain (TD) OCT system using a broadband SLD source. Measurements from all fibres were acquired simultaneously using a CCD camera ${ }^{5}$. Although partially successful, phase drift of OCT fringes between frames was a problem in this relatively slow system. Also, using a camera meant that predigitisation filtering was unavailable, limiting image contrast. Work by a group from California discusses an alternative implementation for a TD-OCT system ${ }^{6}$, in which a bundle forms the sample arm of a fibre Michelson interferometer, and a beam scans across the input end of the bundle. OCT imaging of rabbit trachea was demonstrated with this system.

The swept-source used in our current system, with Fourier-domain processing, removes the requirement for depthscanning, enabling a common-path probe design in which the bundle does not form part of the OCT interferometer. This is advantageous for several reasons. Firstly, it is not possible to control the state of polarisation (SOP) within all (or indeed any) of the individual fibres of a coherent bundle. Thus fringe visibility will vary between fibres, leading to poor contrast in some parts of the image if the bundle resides within the interferometer. Secondly, an imaging bundle has a high NA; fibres can be few-moded under some coupling conditions, leading to ghost images, as reported by Xie et al. ${ }^{6}$. A common path arrangement alleviates both ghosting and problems due to dispersion, including modal dispersion.

\section{TRANSMISSION PROPERTIES OF COHERENT FIBRE BUNDLES}

Standard coherent bundles are intended for use in the visible. These can be few-moded in the near IR under some coupling conditions. Internal losses in bundles are higher than in single fibres; there is the loss of light that impinges on regions outside the fibre cores, and is therefore unguided. There is also some absorption in the fibres. The overall loss for

*h.d.ford@cranfield.ac.uk; Tel. +44 (0)1234 750111; http://www.cranfield.ac.uk/soe/departments/processsystems/engineeringphotonics 
Schott bundles is quoted at around $40 \%$ for a 1 metre bundle in the visible region of the spectrum. The quoted loss typically includes reflections of about $4 \%$ at the proximal and distal polished surfaces, though these can be suppressed by angle-polishing the ends of the guide and, potentially, by application of coatings to the end surfaces.

We are currently using leached imaging bundles supplied by Schott North America ${ }^{7}$. Leached bundles are manufactured

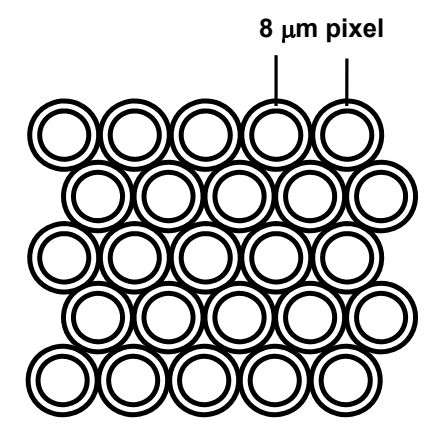
from hexagonally-packed rods, comprising core, cladding and secondary cladding fused into a rigid bar, drawn to achieve the required core diameter, then etching the secondary cladding from the central section to leave a flexible image conduit. The packing arrangement in the $\sim 1 \mathrm{~cm}$ rigid sections at either end is as shown in figure 1. For OCT, optimum imaging wavelengths are in the near infra-red. The transmission of bundle fibres, however, drops off at higher wavelengths and the glass exhibits an inconvenient $\mathrm{OH}$ absorption at around $1400 \mathrm{~nm}^{8}$ which affects the upper end of our laser sweep range, centred at $1330 \mathrm{~nm}$. The overall transmission of a $1350 \mathrm{~mm}$ long bundle, measured with the swept source, was only around $6-8 \%$, including fill-factor and end-reflection losses. Since the beam must traverse the bundle twice in the OCT system, less than $1 \%$ of the initial coupled power is returned to the detector, ignoring other losses in the system. The simplest means to minimise losses is to use a shorter bundle; lengths of about $0.5 \mathrm{~m}$ are readily available. Another possibility is a bundle

Figure 1. Schematic of a leached bundle, showing hexagonal specifically designed for use in the infra-red. These are now offered for sale, though the spectral transmission characteristics for the entire wavelength range of interest are not readily available.

It is also important in OCT that internal cross-coupling between the fibres should be negligible. In the leached bundle used here, $1300 \mathrm{~nm}$ light was well-confined to a single core but, at wavelengths approaching $1550 \mathrm{~nm}$, light focused into one core emerged at the distal end from multiple cores, with the internal crosscoupling worsening as wavelength increased.

\section{BUNDLE-BASED SWEPT-SOURCE OCT PROBE DESIGN}

\subsection{Outline of system}

The OCT probe design, shown in figure 2, incorporates an imaging bundle and a $20 \mathrm{kHz}$ swept laser at $1330 \mathrm{~nm}$ centre wavelength. The system operates in the spectral domain, with a fixed path-length imbalance. Mechanical scanning of the beam takes place at the bundle input end, while mechanical motion within the endoscopic section is avoided. A commonpath configuration has been chosen to avoid loss of visibility due to polarisation mismatches ${ }^{9}$. A miniature Michelson interferometer was constructed at the probe tip, using a broadband 20/80 beamsplitter cube. This achieves a ratio of signal/reference beam powers appropriate for good visibility OCT fringes, without the necessity for attenuation of the reference beam. The side-illumination of the sample allows all the optical elements at the probe tip to be centred on the

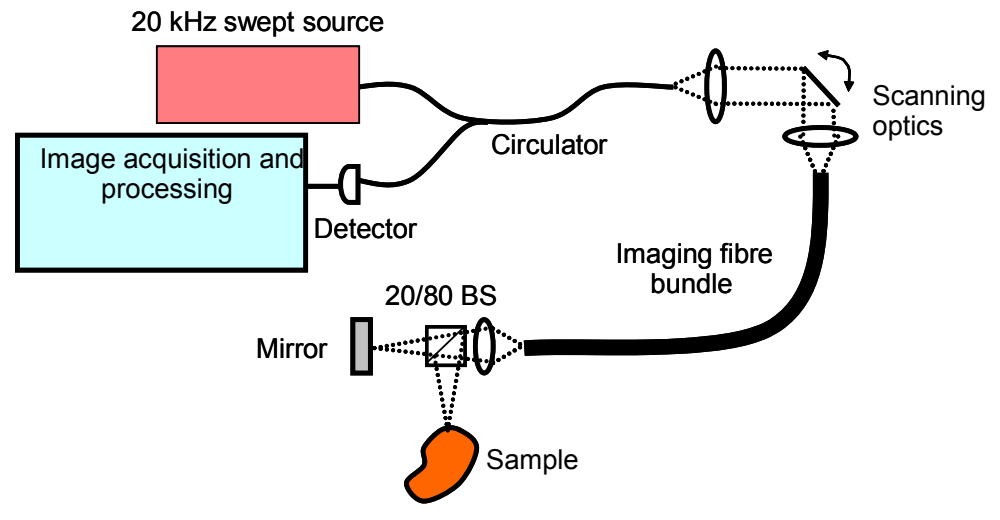

Figure 2. Schematic of common-path FD-OCT system using a Michelson interferometer at the probe tip and incorporating an imaging bundle. optical axis, allowing a small probe diameter to be achieved by the use of appropriate components. Light is coupled from the laser, via a broadband circulator, to the optical bench. At the output fibre, an aspheric lens collimates the beam onto a single-axis galvanometer mirror. This will shortly be upgraded for full 3D acquisition. A second aspheric lens focuses the beam onto the bundle input face, with a beam waist smaller than the fibre core diameter.

The beam is currently scanned rasterfashion at $80 \mathrm{~Hz}$ across the input end of the bundle. A scan rate is selected to ensure a quasi-static beam during the $50 \mu$ duration of the wavelength sweep. Step-and-settle scanning, where the beam is stepped to each

fibre in turn, is also being investigated. The mirror scan is synchronised with acquisition; a digitiser collects several hundred data points in the $\mathrm{z}$ dimension (depth) for each laser sweep. 
The leached imaging bundle has an NA exceeding 0.5. Although the high NA can lead to multi-moding, this is limited by using an input lens with a lower NA. At the probe tip, large NA imaging results in a small beam waist, but a poor Rayleigh range. Reducing the NA improves the depth of focus, but in turn leads to reduced lateral resolution, so a compromise must be reached between the interdependent imaging parameters. Our system operates at present with a magnification of about 2.5. This provides a Rayleigh range of about $100 \mu \mathrm{m}$ with a lateral resolution of about $20-25 \mu \mathrm{m}$. For a probe with unit magnification, the lateral resolution of the instrument is equal to the core spacing. The depth resolution is determined by the sweep range of the laser source. For the $1330 \mathrm{~nm}$ source, with $\sim 100 \mathrm{~nm}$ sweep range, used in this study, a depth resolution of about $8 \mu \mathrm{m}$ in air is expected.

In contrast to a single-fibre OCT probe, the focusing optics must ensure low-loss, low-aberration imaging for off-axis object points. The field of view for the probe lens is typically rather small, since the coherent bundle diameter is unlikely to exceed $2 \mathrm{~mm}$. However, significant care is still required to ensure adequate operation towards the periphery of the images. At present, with a $1 \mathrm{~mm}$ diameter bundle, aspheric lenses result in well-focused images of peripheral fibres but, for larger diameter bundles, coated, multi-element lenses are likely to be the best solution.

Light returning from the OCT probe re-enters the delivery fibre, and is directed by the circulator to one arm of a $15 \mathrm{MHz}$ bandwidth balanced detector. A small amount of source light, routed to the second arm of the balanced detector via a variable optical attenuator, allows much of this stray light to be balanced out, thereby improving OCT contrast.

The output from the detector is passed to a high-speed digitiser card, synchronised both to the laser scan and to the scanning mirror frequency. 800-1500 samples are acquired for each sweep of the laser. The total number of A-scans per raster is currently set to about 200. This ensures that each fibre is sampled at least once, assuming good coupling into all fibres. A Fourier transform is performed on the data set (re-sampling from wavelength into frequency space is required first, to optimise resolution), and the logarithm of the result is calculated to produce a pseudo real-time display $(\sim 5 \mathrm{~Hz})$ of the images. Lateral resolution is ultimately determined by the fibre core separation, and depth resolution by the laser sweep range.

\section{RESULTS AND DISCUSSION}

The system was tested, initially on glass samples and subsequently on samples of vegetable tissue. Results are shown in figure 3. Images obtained from a single-fibre OCT system, of identical configuration to the bundle-based system except that the scanning was moved to the probe tip, are also shown for comparison. It is clear that the SNR, as would be expected, is currently much higher in the single-fibre system. The optical power at the detector is sufficient to obtain near-saturation, even with additional attenuation of the reference beam. Unfortunately this is not the case at present for the bundle-based system, and detector noise dominates.

Images from the bundle-based system were acquired prior to the introduction of re-sampling from wavelength to frequency, hence the lower depth resolution. However, in the image of a microscope cover slip (figure 3(c)) the front and rear surfaces, represented by the top and bottom lines in the image, are clearly visible, separated by about $200 \mu \mathrm{m}$. The intermediate line is due to a self-interference between reflections from the front and rear surfaces of the cover slip, and appears because the reflected intensities for this object are comparable to that of the reference reflection. Unlike the other two lines in the image, this one does not move as the object is translated along the optical axis, because the path-length difference is fixed by the object thickness. Figure 3(d) shows preliminary results from a sample of spring onion. The SNR is low in this image, probably as a result of the very high bundle transmission loss, but two layers can be clearly distinguished, separated by about 100-200 $\mu \mathrm{m}$. Features arising from the bundle characteristics are visible in the lower images. There is a distinctive pixilation, which corresponds to illumination by individual fibres, and a lack of signal in the interstitial 'dead spaces'. Images could be improved by smoothing or, at the expense of a reduction in lateral resolution and power at the detector, by enlarging the beam waist at the bundle input to a diameter exceeding that of the fibre cores. The pixilation is prominent here because the image is formed by a bundle containing a relatively small number of fibres; the number within this scan is about 65. It would be desirable to select a bundle of somewhat greater diameter, containing a larger number of fibres of smaller core diameter. This would improve the data density within the image. Another feature of figure 3(d) is the periodic variation in intensity across the image, attributed to a misalignment between the raster scan and the fibre packing directions. Unless the rows of bundle fibres are perfectly aligned with the raster scan, the coupling will vary periodically as the beam traverses the rows, giving rise to 'patchiness' of the detected signal. This is a problem only for a single transverse scan, and can be removed by acquiring several scans with a lateral offset of a few microns between each, and averaging. When 2D mirror scanning is introduced, smoothing will have the same effect. 


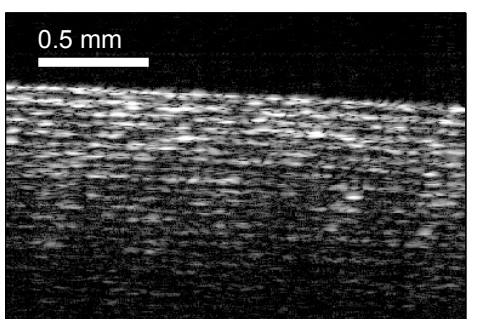

(a)

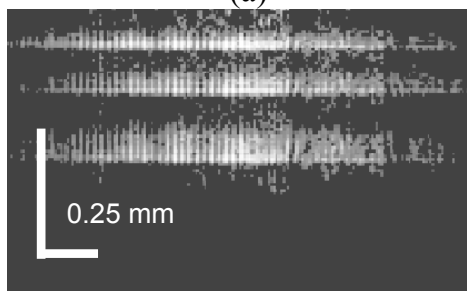

(c)

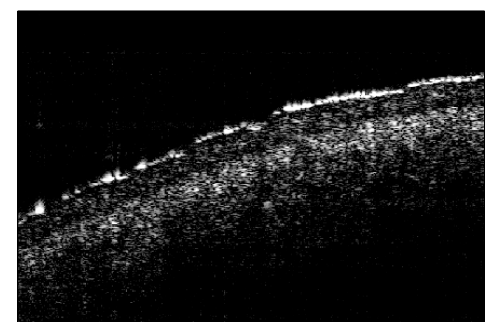

(b)

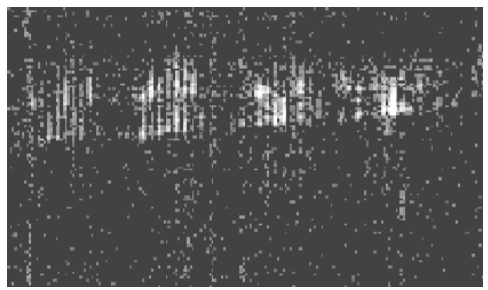

(d)

Figure 3. OCT images. Upper images: (a) reel of scotch tape and (b) skin of hand acquired using a single-fibre OCT system with scanning at probe tip. Scale applies to $x$ and $y$ directions. Lower images: (c) microscope cover slip and (d) spring onion acquired using bundle-based system with scanning at bundle input.

'walk-off' from the reference mirror for large-angle rays. Modification of the optics in the probe-end imaging system will reduce this problem.

\section{ACKNOWLEDGMENTS}

We are grateful for a grant (EP/F034679/1) from the Engineering and Physical Science Research Council, UK.

\section{REFERENCES}

[1] Feldchtein, F. I., Gelikonov, V. M. and Gelikonov, G. V., [Handbook of Optical Coherence Tomography], Informa Health Care, London, New York, Melbourne and Stockholm, 125-143 (2002).

[2] Han, S., Sarunik, M. V., Wu, J., Humayun, M. And Yang, C., "Handheld forward-imaging needle endoscope for ophthalmic optical coherence tomography inspection", Journal of Biomedical Optics Letters, 13(2), no. 020505 (2008).

[3] Tearney, G. J., Brezinski, M. E., Bouma, B. E., Boppart, S. A., Pitris, C., Southern, J. F. and Fujimoto, J. G., "Invivo endoscopic optical biopsy with optical coherence tomography", Science, 276(5321), 2037-2039 (1997).

[4] Xu, Y., Singh, J., Premachandran, C. S., Khairyanto, A., Chen, K.W.S., Chen, N., Sheppard, C. J. R. and Olivo, M., "Design and development of a 3D scanning MEMS OCT probe using a novel SiOB package assembly", J. Micromech. Microeng. 18, no. 125005 (2008).

[5] Ford, H. D. and Tatam, R. P. "Full-field optical coherence tomography", Proc. SPIE 5858-19 (2005).

[6] Xie, T., Mukai, D., Guo, S., Brenner, M. and Chen, Z., "Fibre-optic-bundle-based optical coherence tomography", Opt. Lett. 30(14), 1803-1805, (2005).

[7] http://www.schott.com/fiberoptics/english/products/healthcare/imagingfiberoptics/flexiblecomponents

[8] Gusarov, A., Doyle, D., Glebov, L. and Berghmans, F., "Comparison of radiation-induced transmission degradation of borosilicate crown optical glass from four different manufacturers", Proc. SPIE 5897-01 (2005).

[9] Casaubieilh, P., Ford, H. D., James, S. W. and Tatam, R. P., "Optical coherence tomography with a Fizeau interferometer configuration", Proc. SPIE 5858-18, (2005). 